\title{
Evaluation of Hypertension and Pre-Hypertension among Obese Children Living in Saudi Arabia by National and International Guidelines of Hypertension
}

\author{
Reham Wagdy 1, 2*, Sameer Al-Ostaz ${ }^{2}$, Omar Al-ghamdi ${ }^{2}$, Ahmad Kanaa ${ }^{2}$ Saleh Al-Shareef ${ }^{2}$, \\ Mohammad Al-Mazidii ${ }^{2}$, Ensaf Albokhari ${ }^{2} \&$ Maha Al-Attas ${ }^{2}$. \\ ${ }^{1}$ Department of Pediatrics, Faculty of Medicine, Alexandria University, Alexandria, Egypt, \\ ${ }^{2}$ Medicine Program, Batterjee Medical College for Science and Technology, 6231 Jeddah 21442, Saudi Arabia. \\ *Corresponding author: Reham Wagdy, Mobile: (+20)1005088297 Email:
}

\begin{abstract}
Background: Pediatrics hypertension is a major public health concern. The burden of hypertension among children has increased especially in high-income countries due to the surge in obesity prevalence.

Objective: The current study aimed to evaluate the differences and similarities in the prevalence of hypertension and prehypertension among obese students living in Jeddah estimated by the updated Saudi guidelines and the guidelines of American Academy of Pediatrics for hypertension.

Patients and Methods: This cross-sectional study included a total of 107 students having BMI >18.5 and 192 students having BMI $<18.5$, served as a control, matched in age and gender. They were collected from national and international schools and from out-patient clinics of Hospitals of Ministry of Health. This study was done between October 2019 and March 2020. The participants were subjected to blood pressure measurements, classified based on updated Saudi \& American guidelines of hypertension 2017.

Results: Among overweight/obese students' systolic hypertension was significantly higher and was 2.344 more likely to occur than among normal students $(95 \% \mathrm{CI}: 1.283-4.285 .<0.001)$, the prevalence of systolic hypertension grade I was significantly higher by Saudi guidelines compared to that recorded by American among overweight/obese group ( $21.5 \%$ versus $12.1, \mathrm{P}=0.004)$. The prevalence of elevated systolic blood pressure by American guidelines was significantly higher among overweight/obese group above 13 year when compared to its similar by Saudi guidelines (37.4\% versus $25.2 \%, \mathrm{P}=0.004$ ).

Conclusion: It could be concluded that overweight/obese students are at least two times more susceptible for hypertension than normal body weight students. A fixed threshold for hypertension of the updated American guidelines for teenager could be the cause of a down-estimation of prevalence of hypertension to prehypertension state.
\end{abstract}

Keywords: Hypertension; Pre-hypertension; Obesity; Saudi guidelines of hypertension

\section{INTRODUCTION}

Hypertension is a major public health problem. It is a leading cause of death globally, accounting for about 10.4 million deaths per year ${ }^{(1)}$. In 2010, an estimated 1.39 billion people had hypertension worldwide with adverse impact on cardiovascular and renal morbidity ${ }^{(2)}$. The importance of hypertension among children has not been well appreciated as in adults although presence of hypertension in children usually leads to hypertension in adulthood. In the past, primary hypertension in children was considered a non-common disease. Currently, the incidence of hypertension in children has been increased and widely ranged from $0.3 \%$ up to $21 \%$ among diversity of population ${ }^{(3)}$. Different risk factors for pediatrics hypertension have been studied; genetic factors, ethnic groups, gender, body mass index, physical activity and stress, however, obesity is a main risk ${ }^{(3,4)}$. Evidence showed strong relation of blood pressure (BP) to dyslipidemia, vascular dysfunction, increased left ventricular mass and type 2 Diabetes in obese population ${ }^{(5)}$.
The epidemiological changes of hypertension might be attributed to the growing rates of overweight and obesity among population ${ }^{(6,7)}$.

Childhood obesity is considered by World Health Organization (WHO) a public-health threats for children over the world. Particularly in Saudi Arabia, the prevalence of overweight and obesity is rising alarmingly among children and adolescents over the past decade. The overall prevalence of obesity rate has been doubled over a 10 -year period from $(\approx 9.3 \%)$ as reported in 2004 by the WHO-based national prevalence rate to $18.2 \%$ at 2015 with significant predominance among adolescents (>11 years) ${ }^{(8)}$. Obese children often, become obese adolescents and eventually obese adults ${ }^{(9)}$.

Strong initiatives had been implemented for obesity prevention/intervention programs in Saudi Arabia for Saudi community. Thus, studying prehypertension and hypertension among obese children was challenging ${ }^{(8)}$.

Systemic hypertension, although easy to be clinically measured and assessed, is a silent disease, 
whose degenerative and cumulative effect is greater for younger individuals due to their longer exposure ${ }^{(9)}$.

Saudi Hypertension Management Society (SHMS) has taken up the leading role of spreading the knowledge and care for hypertension. Formulation of the national guidelines of hypertension was an important step and updating the Saudi Guidelines (SGL) was innovative, especially after the release of unique version 2018, classifying hypertension in children ${ }^{(10)}$. Currently, many international guidelines for pediatrics hypertension are available but the Guidline of American Academy of pediatrics (AAP-GL) had been updated lately at $2017^{(11)}$.

The current study was aimed to study the prevalence of hypertension and prehypertension conditions among obese children living in Saudi Arabia by the new Saudi and American guidelines (versions 2017) in order to evaluate the impact of the differences between both guidelines on the disease prevalence.

\section{PATIENTS AND METHODS}

This cross-sectional study included a total of 107 students having BMI $>18.5$ and 192 students having BMI <18.5, served as a control, matched in age and gender. They were collected from national and international schools and from out-patient clinics of Hospitals of Ministry of Health (MOH), who were suffering from mild acute illness as common cold, tonsillitis or acute diarrhea diseases. This study was started at October 2019 and ended by March 2020 because of Covid-19 pandemics.

\section{Ethical Consideration:}

This study was ethically approved by Ethical and Research Committee, Batterjee Medical college at Jeddah, Saudi Arabia No (RES-2019-0005) and ethical approval of Faculty of Medicine, Alexandria University, Egypt (serial number: 0304273).

An informed consent was received from all caregivers. School advocatory helped our team for approaching the students with signed consent.

\section{Sample size:}

Based on the criteria of the current study, the sample size was estimated based on the following assumptions: alpha error $=5 \%$ and study power $=80 \%$. According to Mazor-Aronovitch et al. 17.2\% (25/145) of children with body mass index (BMI) above the 85th percentile had hypertension compared to $1.9 \%$ (2/105) of children with normal weight (BMI less than 85 th percentile) ${ }^{(12)}$. $\operatorname{group}^{(13)}$.

\section{Inclusion criteria:}

The included students were healthy, from both genders, Saudi and non-Saudi, living at Jeddah, KSA and aged between 5 years to 18 years. The students were collected from national, international schools and outpatient clinics of $\mathrm{MOH}$ hospitals. The recruited cases $(n=107)$ had BMI $>18.5$ (or $>85$ th percentiles for age). Meanwhile, the students served as a control group ( $\mathrm{n}=192$ ) had BMI $<18.5$ (or $<85$ th and $>5$ th percentiles for age).

\section{Exclusion criteria:}

Students with chronic diseases, syndromes, underweight and those on chronic medications that might affect blood pressure as corticosteroids were excluded from the study.

Demographic data of the children were checked (age, gender, nationality). Systemic examination was done for all participants with recording anthropometric parameters and blood pressure measurements.

Anthropometric measurements:

All students were examined for recording weight, height, and BMI. Height was measured to the nearest centimeter with a portable stadiometer. Weight was measured to the nearest kilogram using electronic digital scales. Body mass index (BMI) values were calculated using measured height and weight values [weight (kilograms)/height (meter ${ }^{2}$ ). Anthropometric Z-scores were calculated relative to age- and gender. The WHO percentile body mass index "BMI"/age for males and females charts was used to determine the body status as follows ${ }^{(14)}$;

Normal weight: 5 th $-<85$ th percentile, matches in adult $\mathrm{BMI}>18.5$.

Overweight: 85 th $-<95$ th percentile, $\mathrm{Z}$ score above +1 and below +2 SDS, matched in adult BMI $>25$ and $<$ 30.

Obese: > 95th percentile, $\mathrm{Z}$ score above >+2 SDS, matches in adult $\mathrm{BMI}>30$.

\section{$>$ Measurement of the blood pressure:}

Blood pressure was measured three times using a proper pediatric cuff size at rest and in relaxed time in the day (noon). Electronic devices using oscillometer techniques (Omron M3W; HEM-7202-E, OMRON Healthcare Co., Ltd, Kyoto, Japan) were used in the study.

The average records were estimated. Corresponding blood pressure percentiles were determined based on students' height, age and gender for all students according to international standards ${ }^{(15)}$. The current Saudi National Standard Nomogram were not used as they are recommended for updating to 
consider possible differences for some geographic areas of the country ${ }^{(16)}$.

Interpretations of blood pressure systolic or diastolic records for children (5y-18):

According to Saudi Guidelines $2018{ }^{(10)}$

- Normal BP: $<90^{\text {th }}$ percentiles for age, gender and height.
- Prehypertension (Pre-HTN) BP: $>90^{\text {th }}$ percentiles and $<95^{\text {th }}$ percentiles, or $>120 / 80$

- Hypertension (HTN) Grade I: from $95^{\text {th }}$ percentiles till ( $99^{\text {th }}$ percentiles $\left.+5 \mathrm{mmHg}\right)$.

- HTN Grade II: $>99^{\text {th }}$ percentiles $+5 \mathrm{mmHg}$.

According to AAP-GL of hypertension 2017, Table.1; ${ }^{(11)}$

Table 1. Updated definitions of blood pressure categories according to guidelines of American Academy of Pediatrics

\begin{tabular}{|c|c|}
\hline For Children Aged $1-13$ y & For Children Aged $\geq 13$ y \\
\hline Normal BP: $<90$ th percentile & Normal BP: $<120 /<80 \mathrm{~mm} \mathrm{Hg}$ \\
\hline $\begin{array}{l}\text { Elevated BP: } \geq 90 \text { th percentile to }<95 \text { th percentile or } 120 / 80 \\
m m \mathrm{Hg} \text { to }<95 \text { th percentile (whichever is lower) }\end{array}$ & Elevated BP: $120 /<80$ to $129 /<80 \mathrm{~mm} \mathrm{Hg}$ \\
\hline $\begin{array}{l}\text { Stage } 1 \mathrm{HTN}: \geq 95 \text { th percentile to }<95 \text { th percentile }+12 \mathrm{mmHg} \text {, } \\
\text { or } 130 / 80 \text { to } 139 / 89 \mathrm{~mm} \mathrm{Hg} \text { (whichever is lower) }\end{array}$ & Stage $1 \mathrm{HTN}: 130 / 80$ to $139 / 89 \mathrm{~mm} \mathrm{Hg}$ \\
\hline $\begin{array}{l}\text { Stage } 2 \mathrm{HTN}: \geq 95 \text { th percentile }+12 \mathrm{~mm} \mathrm{Hg} \text {, or } \geq 140 / 90 \mathrm{~mm} \mathrm{Hg} \\
\text { (whichever is lower) }\end{array}$ & Stage 2 HTN: $\geq 140 / 90 \mathrm{~mm} \mathrm{Hg}$ \\
\hline
\end{tabular}

\section{Statistical analysis of the data:}

Data were fed to the computer and analyzed using IBM SPSS software package version 20.0. Data were fed to the computer and analyzed using IBM SPSS software package version 20.0. (Armonk, NY: IBM Corp). The Kolmogorov- Smirnov, Shapiro and D'agstino tests were used to verify the normality of distribution of variables, Comparisons between groups for categorical variables were assessed using Chisquare test. Student t-test and ANOVA test were used to compare normally distributed quantitative variables. Mann Whitney test was used to compare between two groups for abnormally distributed quantitative variables. Significance of the obtained results was judged at the 5\% level.

\section{RESULTS}

\section{Students characteristics:}

This is a cross- sectional study conducted over 299 students living in Jeddah Saudi Arabia, included $51.8 \%$ male and $48.2 \%$ female, aged between $5 \mathrm{y}$ and $17 \mathrm{y}$ with mean age of $11.5 \pm 2.7 \mathrm{y}$. About $69.2 \%$ of the children were Saudi students while $30.8 \%$ were of mixed nationality. According to BMI, the study group included $107(35.8 \%)$ overweight/obese students which were matched in age and gender with the other 192 normal weight students (64.2\%) who had been considered as controls. Students with high BMI were distributed as following; Overweight $61.7 \%$ and obese $37.3 \%$ (class I; $29.9 \%$, class II: $8.4 \%$ ).

Table 2 shows the patients characteristics of the three subgroups normal $(64.2 \%)$, overweight (20\%) and obese group (13.7\%). The females were significantly predominating in overweight group compared to obese group (53\% versus $24.4 \%$, $\mathrm{p} 1=0.004)$. The prevalence of males was significantly higher in obese group compared to normal students. The percent of overweight and obese children were significantly higher among adolescents (above 13 year) when compared to controls of similar age group $(56.1 \%$ \& $75.6 \%$, respectively versus $38.5 \%$, P1:0.00.5, P2:<0.001). The mean of weight, height and BMI were significantly higher for obese groups when compared to overweight or controls as shown in Table 2.

\section{Characteristics of pre-hypertension \& hypertension among study group by S-GL \& AAP-GL}

The overall characteristics of blood pressure records, percentiles, and prevalence of hypertension of the normal weight, overweight and obese subgroups according to S-GL and AAP-GL were summarized in Table 3. The prevalence of systolic prehypertension among the sample of the study was $17.7 \%$ while systolic HTN-I was $16.1 \%$ and HTN-II was $3.7 \%$. The prevalence of diastolic pre-HTN was $20.4 \%$, diastolic HTN-I was $15.4 \%$ and diastolic HTN-II was less 3.7 $\%$. The mean of systolic BP records was higher for obese students with significant difference compared to overweight or controls, respectively ( $123.6 \pm 8.6$ versus $116.2 \pm 8.8$ or $111.9 \pm 7.7,<0.001)$. Whereas the mean of diastolic BP did not differ significantly between three subgroups. According to Saudi guidelines, the percent of systolic prehypertension and or systolic hypertensive students grade I were significantly higher among obese group when compared to overweight and or the controls, respectively $(36.6 \% \& 31.7 \%$ versus 
$18.2 \% \quad \& 15.2 \%$ and $13 \% \quad \& 13.5 \%, \mathrm{p} 1=0.004 \&$ P3<0.001). Similarly, the percent of elevated systolic BP and systolic hypertension stage I findings by (AAP-GL) were significantly higher in obese groups when compared to other groups $(48.8 \%$ \& $22 \%$ versus $30.3 \% \& 6.1 \%$ and $17.2 \% \& 11.5 \%, \mathrm{p} 1=0.003 \&$ $\mathrm{P} 3<0.001)$. The percent of diastolic hypertension students did not differ significantly between the three subgroups whether by SGL or AAP.

Table 4 compared the prevalence of hypertension by both guidelines between high BMI students (overweight/obese: $\mathrm{n}=107$ ) and normal BMI students (controls: $n=192$ ). Through S-GL, the prevalence of systolic pre-HTN among overweight/obese was higher with significant difference when compared to controls (25\% versus $13 \%, \mathrm{P} ;<0.001)$. At Confidence of $95 \%$, systolic PreHTN can occur 2.69 times more likely among high BMI students $(1.44-5.04)$ rather than normal BMI. Additionally, systolic HTN was significantly higher among high BMI group, and was 2.344 times more likely to occur among cases $(1.283-4.285$. <0.001) when compared to controls as shown in Table 4.

On the other side, the combined prevalence of Pre-HTN and HTN among all participants was (56.6\%; $29.5 \%$ for pre-HTN \& $27.4 \%$ for HTN). Meanwhile, The combined prevalence of Pre-HTN and HTN among overweight/obese subgroup was $70 \% ; 39.2 \%$ \& $30.8 \%$, respectively) by S-GL.

In addition, the relation between both guidelines was studied. The prevalence of elevated systolic BP by AAP-GL was less than the prevalence of systolic pre-hypertension by S-GL by (11\%). The $\kappa$-statistic was good (0.72) with overall agreement of both guidelines was $88.9 \%$. Meanwhile, systolic hypertension grade I prevalence by S-GL was significantly higher compared to the recorded prevalence of systolic hypertension stage I by AAPGL $(<0.001)$ as summarized in Table 5-A. The prevalence of stage 1 diastolic hypertension by American Academy of Pediatrics (AAP-GL) was slightly less than S-GL. The $\kappa$-statistic was good (0.66) with overall agreement of $85.5 \%$. The prevalence of diastolic norm tension students by APP-GL was in $100 \%$ agreement to Saudi guidelines with good $\kappa$ statistic 0.662. However, Prevalence of diastolic prehypertension students by S-GL was significantly higher than AAP-GL as shown in Table 5-B.
The prevalence of systolic hypertension grade I was significantly higher by Saudi Guidelines compared to American (21.5\% versus 12.1, $\mathrm{P}=0.004)$. Meanwhile, the prevalence of systolic elevated blood pressure by American guidelines was significantly higher compared to prevalence of systolic prehypertension by Saudi (37.4\% versus 25.2\%, $\mathrm{P}=0.004)$ as summarized in Table 6.

\section{Characteristics of pre-hypertensive and hypertensive students among overweight/obese.}

Some risk factors of hypertension among overweight/ obese children were studied to evaluate its effect on hypertension classification by both guidelines as age, gender, and nationality.

High BMI students were classified into three age groups as shown in Tables $2 \& 7$. Although the highest percent of systolic pre-HTN was found among adolescent $(>13 y)$, it showed no significant difference when compared to other age groups. Similarly, the highest percent of systolic HTN- grade I was found among young students $(5 \mathrm{y}-8 \mathrm{y})$ but with insignificant difference to other age groups. However, the prevalence diastolic HTN-I by S-GL and AAP-GL was significantly higher among students between 9y and $12 \mathrm{y}$ when compared to adolescents or young students (18.2\% versus $11.8 \%$ \& $0 \%, \mathrm{P}: 0.010)$ and $(39.3 \%$ versus $11.8 \%, 0 \%, \mathrm{P}:<0.001)$ respectively, as summarized in Table 7. Concerning obese students older than 13 years, the prevalence of systolic elevated BP by AAP-GL was higher with significant difference than the Pre-HTN by S-GL. On other side, systolic HTN-grade I was higher by S-GL with significant difference when compared to its similar by AAP-GL as summarized by Table 8 . There was no significant difference between the prevalence of pre-HTN and or HTN among the males and females of this group $(21 \%$ $\& 32.2 \%$ versus $22.2 \% \& 36.7 \%$, respectively ${ }^{\mathrm{MC}} \mathrm{p}=$ $0.195)$.

As regard the nationality, prevalence of systolic pre-HTN and HTN among Saudi students were higher when compared to non-Saudi students with no statistically significant difference $(26.3 \%$ and $28.2 \%$ versus $22.2 \& 18.8 \%$ ) as shown in Table 9. Similar results were found regarding the diastolic pressure records. 
Table 2. Demographic characteristics and anthropometric measurements of the studied subgroups

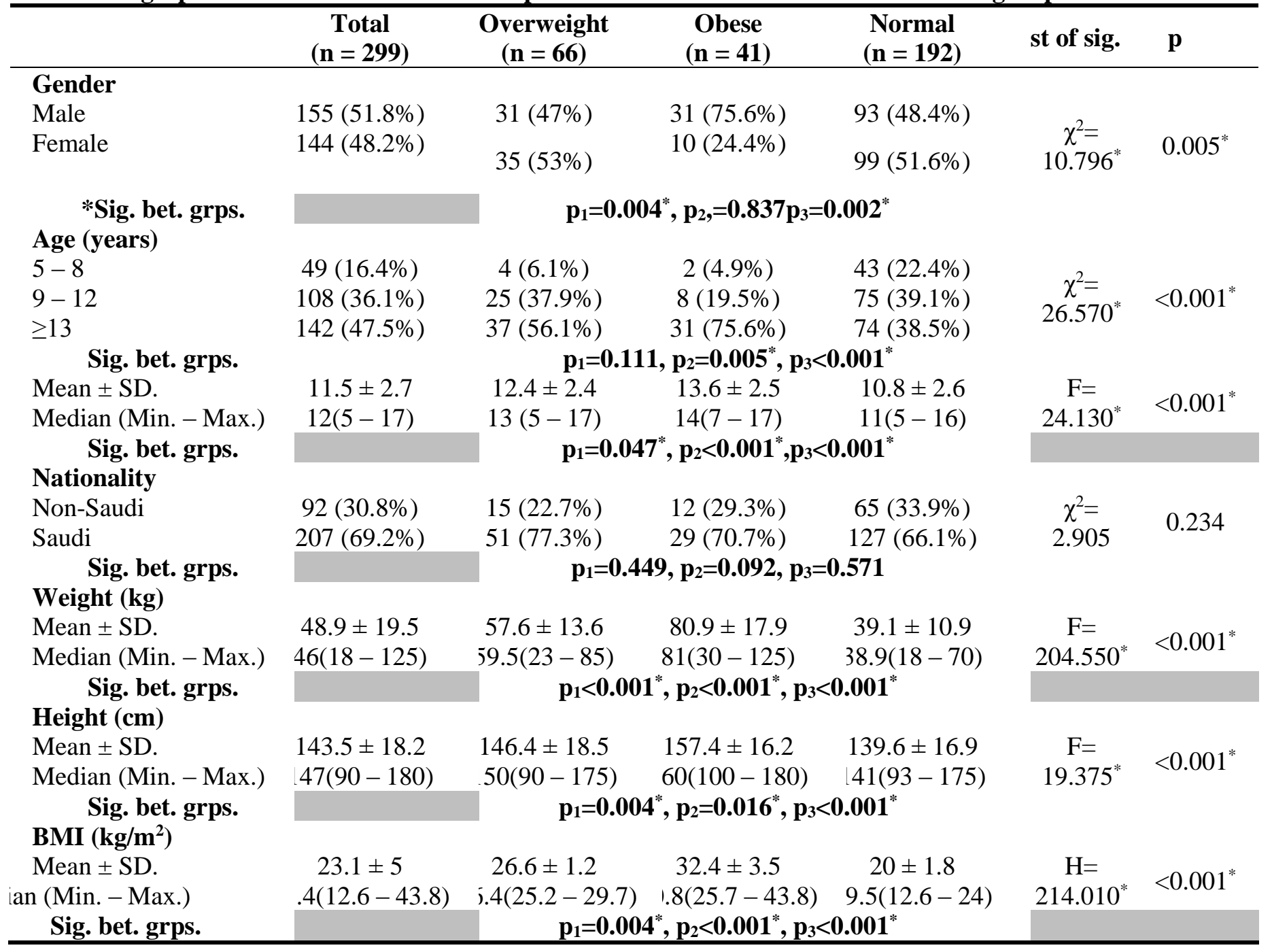

\section{$\chi^{2}$ : Chi square test}

F: F for ANOVA test, Pairwise comparison bet. each 2 groups was done using Post Hoc Test (Tukey)

H: H for Kruskal Wallis test, Pairwise comparison bet. each 2 groups was done using Post Hoc Test (Dunn's for multiple comparisons test)

$\mathrm{p}$ : $\mathrm{p}$ value for comparing between the studied groups

$\mathrm{p}_{1}$ : $\mathrm{p}$ value for comparing between overweight and obese

$\mathrm{p}_{2}: \mathrm{p}$ value for comparing between overweight and non-obese

$\mathrm{p}_{3}: \mathrm{p}$ value for comparing between obese and non-obese.

*: Statistically significant at $\mathrm{p} \leq 0.05$

*Sig. bet. grps: significance between groups 
Table 3. Characteristics of blood pressure measurements of the normal, overweight, and obese students according to Saudi \& American Guidelines

\begin{tabular}{|c|c|c|c|c|c|c|}
\hline & $\begin{array}{c}\text { Total } \\
(n=299)\end{array}$ & $\begin{array}{c}\text { Overweight } \\
(n=66)\end{array}$ & $\begin{array}{c}\text { Obese } \\
(n=41)\end{array}$ & $\begin{array}{l}\text { Normal } \\
(\mathrm{n}=192)\end{array}$ & st of sig. & $\mathbf{p}$ \\
\hline \multicolumn{7}{|l|}{ Systolic BP } \\
\hline Mean \pm SD & $114.4 \pm 9$ & $116.2 \pm 8.8$ & $123.6 \pm 8.6$ & $111.9 \pm 7.7$ & $\mathrm{~F}=$ & \multirow{2}{*}{$0.001^{*}$} \\
\hline Median (Min. - Max.) & $15(95-144)$ & $17(97-144)$ & $25(100-137)$ & $11(95-134)$ & $7.434^{*}$ & \\
\hline Sig. bet. grps. & \multicolumn{6}{|c|}{$p_{1}<0.001^{*}, p_{2}=0.001^{*}, p_{3}<0.001^{*}$} \\
\hline Systolic percentile & & & & & & \multirow{3}{*}{$0.010^{*}$} \\
\hline Mean \pm SD & $78.1 \pm 17.4$ & $79.5 \pm 16.7$ & $85 \pm 16.9$ & $76.2 \pm 17.4$ & $\mathrm{~F}=$ & \\
\hline Median (Min. - Max.) & $83(22-100)$ & $84(31-100)$ & $91(39-100)$ & $79(22-100)$ & $1.664^{*}$ & \\
\hline Sig. bet. grps. & \multicolumn{6}{|c|}{$p_{1}=0.247, p_{2}=0.371, p_{3}=0.009^{*}$} \\
\hline \multicolumn{7}{|l|}{ Saudi GL for systolic } \\
\hline Normal & $187(62.5 \%)$ & $41(62.1 \%)$ & $11(26.8 \%)$ & $135(70.3 \%)$ & \multirow{4}{*}{$\begin{array}{c}\chi^{2}= \\
8.194^{*}\end{array}$} & \multirow{4}{*}{$0.001^{*}$} \\
\hline Prehypertension & $53(17.7 \%)$ & $12(18.2 \%)$ & $15(36.6 \%)$ & $26(13.5 \%)$ & & \\
\hline Hypertension class I & $48(16.1 \%)$ & $10(15.2 \%)$ & $13(31.7 \%)$ & $25(13 \%)$ & & \\
\hline Hypertension class II & $11(3.7 \%)$ & $3(4.5 \%)$ & $2(4.9 \%)$ & $6(3.1 \%)$ & & \\
\hline Sig. bet. grps. & \multicolumn{6}{|c|}{$p_{1}=0.004^{*}, p_{2}=0.648, p_{3}<0.001^{*}$} \\
\hline \multicolumn{7}{|c|}{ AAP GL for systolic BP } \\
\hline Normal & $181(60.5 \%)$ & $39(59.1 \%)$ & $10(24.4 \%)$ & $132(68.8 \%)$ & & \multirow{4}{*}{$0.001^{*}$} \\
\hline Elevated & $73(24.4 \%)$ & $20(30.3 \%)$ & $20(48.8 \%)$ & $33(17.2 \%)$ & $\chi^{2}=$ & \\
\hline Hypertension class I & $35(11.7 \%)$ & $4(6.1 \%)$ & $9(22 \%)$ & $22(11.5 \%)$ & $\begin{array}{ll}\lambda- \\
3.039^{*}\end{array}$ & \\
\hline Hypertension class II & $10(3.3 \%)$ & $3(4.5 \%)$ & $2(4.9 \%)$ & $5(2.6 \%)$ & & \\
\hline Sig. bet. grps. & \multicolumn{6}{|c|}{$p_{1}=0.003^{*}, p_{2}=0.078, p_{3}<0.001^{*}$} \\
\hline Diastolic BP & & & & & & \multirow{3}{*}{0.887} \\
\hline Mean \pm SD & $73.2 \pm 8.1$ & $72.8 \pm 7.2$ & $73.1 \pm 10.1$ & $73.4 \pm 8$ & $\mathrm{~F}=$ & \\
\hline Median (Min. - Max.) & $74(54-96)$ & $74.5(55-85)$ & $71(54-93)$ & $74(54-96)$ & 0.120 & \\
\hline \multicolumn{7}{|l|}{ Diastolic percentile } \\
\hline Mean \pm SD & $80.1 \pm 17.7$ & $77.8 \pm 18$ & $73.7 \pm 23.5$ & $82.2 \pm 15.8$ & $\mathrm{H}=$ & \multirow{2}{*}{0.126} \\
\hline Median (Min. - Max.) & $85(18-100)$ & $34.5(26-99)$ & $82(18-100)$ & $86(28-100)$ & 4.137 & \\
\hline \multicolumn{7}{|l|}{ Saudi GL for diastolic } \\
\hline \multicolumn{7}{|l|}{ BP } \\
\hline Normal & $181(60.5 \%)$ & $40(60.6 \%)$ & $26(63.4 \%)$ & $115(59.9 \%)$ & & \multirow{4}{*}{0.265} \\
\hline Prehypertension & $61(20.4 \%)$ & $19(28.8 \%)$ & $6(14.6 \%)$ & $36(18.8 \%)$ & $\chi^{2}=$ & \\
\hline Hypertension class I & $46(15.4 \%)$ & $7(10.6 \%)$ & $7(17.1 \%)$ & $32(16.7 \%)$ & 7.650 & \\
\hline Hypertension class II & $11(3.7 \%)$ & $0(0 \%)$ & $2(4.9 \%)$ & $9(4.7 \%)$ & & \\
\hline Sig. bet. grps. & \multicolumn{6}{|c|}{$p_{1}=0.090, p_{2}=0.085, p_{3}=0.942$} \\
\hline \multicolumn{7}{|l|}{$\begin{array}{l}\text { AAP GL for diastolic } \\
\text { BP }\end{array}$} \\
\hline Normal & $199(66.6 \%)$ & $47(71.2 \%)$ & $27(65.9 \%)$ & $125(65.1 \%)$ & & \multirow{4}{*}{0.307} \\
\hline Elevated & $27(9 \%)$ & $6(9.1 \%)$ & $2(4.9 \%)$ & $19(9.9 \%)$ & $\chi^{2}=$ & \\
\hline Hypertension class I & $61(20.4 \%)$ & $13(19.7 \%)$ & $8(19.5 \%)$ & $40(20.8 \%)$ & 6.899 & \\
\hline Hypertension class II & $12(4 \%)$ & $0(0 \%)$ & $4(9.8 \%)$ & $8(4.2 \%)$ & & \\
\hline Sig. bet. grps. & \multicolumn{6}{|c|}{$\mathrm{p}_{1}=0.077, \mathrm{p}_{2}=\mathbf{0 . 0 3 7 8 ,}, \mathrm{p}_{3}=0.388$} \\
\hline
\end{tabular}

$\chi^{2}$ : Chi square test

F: F for ANOVA test, Pairwise comparison bet. each 2 groups was done using Post Hoc Test (Tukey)

H: H for Kruskal Wallis test, pairwise comparison bet. each 2 groups were done using Post Hoc Test (Dunn's for multiple comparisons test)

$\mathrm{p}$ : $\mathrm{p}$ value for comparing between the studied groups

$\mathrm{p}_{1}: \mathrm{p}$ value for comparing between overweight and obese

$\mathrm{p}_{2}$ : $\mathrm{p}$ value for comparing between overweight and non-obese

$\mathrm{p}_{3}$ : $\mathrm{p}$ value for comparing between obese and non-obese

Sig. bet. grps: significance between groups 
Table 4. Comparison between the distribution of prehypertension and hypertension between the normal BMI students and High BMI students (overweight/ obese) according to Saudi \& American Guidelines

\begin{tabular}{|c|c|c|c|c|c|}
\hline & weight/obese $(\mathrm{n}=$ & $\begin{array}{c}\text { Normal } \\
(n=192)\end{array}$ & $\chi^{2}$ & $\mathbf{p}$ & OR $(95 \%$ C.I $)$ \\
\hline \multicolumn{6}{|c|}{ Saudi GL for systolic } \\
\hline Normal ${ }^{\circledR}$ & $52(48.6 \%)$ & $135(70.3 \%)$ & - & - & 1.000 \\
\hline Prehypertension & $27(25.2 \%)$ & $26(13.5 \%)$ & 9.721 & $0.001^{*}$ & $2.69(1.44-5.04)$ \\
\hline Hypertension & $28(26.2 \%)$ & $31(16.5)$ & $5.579^{*}$ & $0.001^{*}$ & $344(1.283-4.285)$ \\
\hline \multicolumn{6}{|c|}{ American GL for systolic } \\
\hline Normal ${ }^{\circledR}$ & $49(45.8 \%)$ & $132(68.8 \%)$ & - & - & 1.000 \\
\hline Elevated & $40(37.4 \%)$ & $33(17.2 \%)$ & 7.625 & $0.001^{*}$ & $265(1.854-5.748)$ \\
\hline Hypertension & $18(16.8 \%)$ & $27(14 \%)$ & $.070^{*}$ & $1.024^{*}$ & $795(0.909-3.547)$ \\
\hline \multicolumn{6}{|c|}{ Saudi GL for diastolic } \\
\hline Normal ${ }^{\circledR}$ & $66(61.7 \%)$ & $115(59.9 \%)$ & - & - & 1.000 \\
\hline Prehypertension & $25(23.4 \%)$ & $36(18.8 \%)$ & 0.811 & 0.368 & $.210(0.668-2.19)$ \\
\hline Hypertension & $16(15 \%)$ & $41(21.4 \%)$ & 2.530 & 0.112 & $680(0.354-1.305)$ \\
\hline \multicolumn{6}{|c|}{ American GL for diastolic } \\
\hline Normal ${ }^{\circledR}$ & $74(69.2 \%)$ & $125(65.1 \%)$ & - & - & 1.000 \\
\hline Elevated & $8(7.5 \%)$ & $19(9.9 \%)$ & 0.764 & 0.382 & $711(0.296-1.705)$ \\
\hline Hyperetention & $25(23.4 \%)$ & $48(25 \%)$ & 0.426 & 0.514 & $879(0.510-1.544)$ \\
\hline
\end{tabular}

$\chi^{2}$ : Chi square test, $\mathrm{p}: \mathrm{p}$ value for comparing between the studied groups.

Table 5 (A \& B). Relation between Saudi GL and AAP-GL in classification of systolic \& diastolic hypertension among high BMI students (overweight/obese) group $(\mathbf{n}=107)$

\begin{tabular}{|c|c|c|c|c|c|c|}
\hline \multirow[b]{2}{*}{ Systolic BP } & \multicolumn{4}{|c|}{ Saudi GL } & \multirow[b]{2}{*}{$\square^{\square}$} & \multirow[b]{2}{*}{${ }^{\mathrm{MC}} \mathbf{p}$} \\
\hline & $\begin{array}{l}\text { Normal } \\
(n=52)\end{array}$ & $\begin{array}{c}\text { Pre } \\
\text { pertension } \\
(\mathbf{n}=\mathbf{2 7})\end{array}$ & $\begin{array}{l}\text { ertension cla } \\
\qquad \begin{array}{c}\text { I } \\
(\mathbf{n}=\mathbf{2 3})\end{array}\end{array}$ & $\begin{array}{l}\text { sypertension } \\
\text { class II } \\
(\mathbf{n}=\mathbf{5})\end{array}$ & & \\
\hline \multicolumn{7}{|l|}{ American GL } \\
\hline Normal & $3(92.3 \%)$ & $1(3.7 \%)$ & $0(0 \%)$ & $0(0 \%)$ & \multirow{4}{*}{$38.944^{*}$} & \multirow{4}{*}{0.001} \\
\hline Elevated & t $(7.7 \%)$ & $24(88.9 \%)$ & $12(52.2 \%)$ & $0(0 \%)$ & & \\
\hline Hypertension class I & $0(0 \%)$ & $2(7.4 \%)$ & $11(47.8 \%)$ & $0(0 \%)$ & & \\
\hline \multirow{2}{*}{$\begin{array}{l}\text { Hypertension class II } \\
\boldsymbol{\kappa} \text { (Level of agreement) }\end{array}$} & $0(0 \%)$ & $0(0 \%)$ & $0(0 \%)$ & $5(100 \%)$ & & \\
\hline & \multicolumn{4}{|c|}{$0.729\left(<0.001^{*}\right)$ Good agreement } & & \\
\hline \multicolumn{7}{|c|}{ Saudi GL } \\
\hline Diastolic BP & $\begin{array}{l}\text { Normal } \\
(n=66)\end{array}$ & $\begin{array}{c}\text { Pre } \\
\text { pertension } \\
(\mathbf{n}=\mathbf{2 5})\end{array}$ & $\begin{array}{l}\text { ypertension } \\
\text { class I } \\
(\mathbf{n}=14)\end{array}$ & $\begin{array}{l}\text { ypertension } \\
\text { class II } \\
(\mathrm{n}=2)\end{array}$ & $\square^{\square}$ & ${ }^{\mathrm{MC}} \mathbf{p}$ \\
\hline \multicolumn{7}{|l|}{ American GL } \\
\hline Normal & $6(100 \%)$ & $8(32 \%)$ & $0(0 \%)$ & $0(0 \%)$ & \multirow{4}{*}{$10.906^{*}$} & \multirow{4}{*}{$0.001^{*}$} \\
\hline Elevated & $0(0 \%)$ & $8(32 \%)$ & $0(0 \%)$ & $0(0 \%)$ & & \\
\hline Hypertension class I & $0(0 \%)$ & $9(36 \%)$ & $12(85.7 \%)$ & $0(0 \%)$ & & \\
\hline Hypertension class II & $0(0 \%)$ & $0(0 \%)$ & $2(14.3 \%)$ & $2(100 \%)$ & & \\
\hline $\begin{array}{l}* * \kappa \text { (Level of } \\
\text { agreement) }\end{array}$ & \multicolumn{4}{|c|}{$0.665\left(<0.001^{*}\right)$ Good agreement } & & \\
\hline
\end{tabular}

$\chi^{2}$ : Chi square test MC: Monte Carlo test

$\mathrm{p}$ : $\mathrm{p}$ value for comparing between the studied groups, *: Statistically significant at $\mathrm{p} \leq 0.05$

\begin{tabular}{cc}
\hline$* *$ Value of $\boldsymbol{k}$ & trength of agreement \\
\hline$<0.20$ & Poor \\
$0.21-0.40$ & Fair \\
$0.41-0.60$ & Moderate \\
$0.61-0.80$ & Good \\
-1.00 & Very good \\
\hline
\end{tabular}


Table 6. Comparison between the distribution of prehypertension and hypertension by Saudi GL and by AAPGL among the overweight/obese students $(n=107)$

\begin{tabular}{lcrrrr} 
& Blood pressure & Saudi GL & American GL & $\chi^{\mathbf{2}}$ & ${ }^{\mathbf{M H}_{\mathbf{p}}}$ \\
\cline { 2 - 6 } Systolic & Normal & $52(48.6 \%$ & $49(45.8 \%)$ & & \\
& Prehypertension /Elevated & $27(25.2 \%$ & $40(37.4 \%)$ & $2.886^{*}$ & $0.004^{*}$ \\
& Hypertension class I & $23(21.5 \%$ & $13(12.1 \%)$ & & \\
\hline \multirow{5}{*}{ Diastolic } & Hypertension class II & $5(4.7 \%)$ & $5(4.7 \%)$ & & \\
& Normal & $66(61.7 \%$ & $74(69.2 \%)$ & & \\
& Prehypertension & $25(23.4 \%$ & $8(7.5 \%)$ & $3.814^{*}$ & $<0.001^{*}$ \\
& Hypertension class I & $14(13.1 \%$ & $21(19.6 \%)$ & & \\
\cline { 2 - 6 } & Hypertension class II & $2(1.9 \%)$ & $4(3.7 \%)$ & & \\
\hline
\end{tabular}

\section{MH: Marginal Homogeneity test}

$\mathrm{p}$ : $\mathrm{p}$ value for comparing between Saudi and American , *: Statistically significant at $\mathrm{p} \leq 0.05$

Table 7. Relation and distribution of prehypertension and hypertension according to the age group among overweight/obese students $(\mathbf{n}=107)$

\begin{tabular}{lccccc}
\hline & $\mathbf{5}-\mathbf{8}(\mathbf{n = 6})$ & $\mathbf{9}-\mathbf{1 2}(\mathbf{n = 3 3})$ & $\mathbf{3}(\mathbf{n = 6 8})$ & $\chi^{\mathbf{2}}$ & $\mathbf{p}$ \\
\hline Saudi GL for systolic BP & & & & & \\
Normal & $4(66.7 \%)$ & $17(51.5 \%)$ & $1(45.6 \%)$ & & \\
Prehypertension & $0(0 \%)$ & $8(24.2 \%)$ & $7(27.9 \%)$ & 5.273 & 0.486 \\
Hypertension class I & $2(33.3 \%)$ & $5(15.2 \%)$ & $5(23.5 \%)$ & & \\
Hypertension class II & $0(0 \%)$ & $3(9.1 \%)$ & $2(2.9 \%)$ & & \\
AAP GL for systolic BP & & & & & \\
Normal & $4(66.7 \%)$ & $15(45.5 \%)$ & )$(44.1 \%)$ & & \\
Elevated & $1(16.7 \%)$ & $12(36.4 \%)$ & $7(39.7 \%)$ & 3.923 & 0.678 \\
Hypertension class I & $1(16.7 \%)$ & $3(9.1 \%)$ & $1(13.2 \%)$ & & \\
Hypertension class II & $0(0 \%)$ & $3(9.1 \%)$ & $2(2.9 \%)$ & & \\
Saudi GL diastolic BP & & & & \\
Normal & $3(50 \%)$ & $14(42.4 \%)$ & $7(72.1 \%)$ & & \\
Prehypertension & $2(33.3 \%)$ & $12(36.4 \%)$ & $1(16.2 \%)$ & $4.676^{*}$ & \multirow{2}{*}{$.010^{*}$} \\
Hypertension class I & $0(0 \%)$ & $6(18.2 \%)$ & $(11.8 \%)$ & & \\
Hypertension class II & $1(16.7 \%)$ & $1(3 \%)$ & $0(0 \%)$ & & \\
AAP GL for diastolic BP & & & & & \\
Normal & $3(50 \%)$ & $14(42.4 \%)$ & $7(83.8 \%)$ & & \\
Elevated & $2(33.3 \%)$ & $5(15.2 \%)$ & $1(1.5 \%)$ & $7.487^{*}$ & $0.001^{*}$ \\
Hypertension class I & $0(0 \%)$ & $13(39.4 \%)$ & $(11.8 \%)$ & & \\
Hypertension class II & $1(16.7 \%)$ & $1(3 \%)$ & $2(2.9 \%)$ & & \\
\hline
\end{tabular}

$\chi^{2}$ : Chi square test $, \mathrm{p}: \mathrm{p}$ value for association between different categories $*$ : Statistically significant at $\mathrm{p} \leq 0.05$.

Table 8. Comparison of the distribution of prehypertension and hypertension among overweight/obese students age $\geq 13(n=68)$ by Saudi-GL versus AAP-GL

\begin{tabular}{llcccc}
\multirow{5}{*}{ Systolic } & \multicolumn{1}{c}{ Blood pressure } & Saudi GL & American GL & $\chi^{\mathbf{2}}$ & ${ }^{\mathbf{M H}} \mathbf{p}$ \\
\cline { 2 - 6 } & Normal & $31(45.6 \%)$ & $30(44.1 \%)$ & & \\
& Prehypertension /Elevated & $19(27.9 \%)$ & $27(39.7 \%)$ & 0.201 & \multirow{2}{*}{$0.044^{*}$} \\
& Hypertension class I & $16(23.5 \%)$ & $9(13.2 \%)$ & 8 & \\
& Hypertension class II & $2(2.9 \%)$ & $2(2.9 \%)$ & & \\
& Normal & $49(72.1 \%)$ & $57(83.8 \%)$ & & \\
Diastolic & $11(16.2 \%)$ & $1(1.5 \%)$ & 2.871 & $0.004^{*}$ \\
& Prehypertension & $8(11.8 \%)$ & $8(11.8 \%)$ & & \\
& Hypertension class I & $0(0 \%)$ & $2(2.9 \%)$ & & \\
& Hypertension class II & & &
\end{tabular}

MH: Marginal Homogeneity test

$\mathrm{p}$ : $\mathrm{p}$ value for comparing between Saudi and American

$*$ : Statistically significant at $\mathrm{p} \leq 0.05, *$ : Statistically significant at $\mathrm{p} \leq 0.05$ 
Table 9. The relation and distribution of prehypertension and hypertension between Saudi and non-Saudi students among overweight/obese students by SGL and AAP -GL

\begin{tabular}{|c|c|c|c|c|}
\hline & \multicolumn{2}{|c|}{ Nationality } & \multirow[b]{2}{*}{$\chi^{2}$} & \\
\hline & $\begin{array}{c}\text { Non-Saudi } \\
(\mathbf{n}=\mathbf{2 7})\end{array}$ & $\begin{array}{c}\text { Saudi } \\
(\mathbf{n}=\mathbf{8 0})\end{array}$ & & \\
\hline \multicolumn{5}{|c|}{ Saudi GL for systolic } \\
\hline Normal ${ }^{\circledR}$ & $16(59.3 \%)$ & $36(45 \%)$ & \multirow{4}{*}{1.782} & \multirow{3}{*}{0.410} \\
\hline Prehypertension & $6(22.2 \%)$ & $21(26.3 \%)$ & & \\
\hline Hypertension & $5(18.5 \%)$ & $23(28.8 \%)$ & & \\
\hline \multicolumn{4}{|c|}{ American GL for systolic } & \\
\hline Normal & $15(55.6 \%)$ & $34(42.5 \%)$ & \multirow{3}{*}{1.419} & \multirow{3}{*}{0.492} \\
\hline Elevated & $8(29.6 \%)$ & $32(40 \%)$ & & \\
\hline Hypertension & $4(14.8 \%)$ & $14(17.5 \%)$ & & \\
\hline \multicolumn{5}{|c|}{ Saudi GL for diastolic } \\
\hline Normal & $18(66.7 \%)$ & $48(60 \%)$ & \multirow{4}{*}{0.522} & \multirow{3}{*}{0.770} \\
\hline Prehypertension & $6(22.2 \%)$ & $19(23.8 \%)$ & & \\
\hline Hypertension & $3(11.1 \%)$ & $13(16.3 \%)$ & & \\
\hline \multicolumn{4}{|c|}{ American GL for diastolic } & \\
\hline Normal & $20(74.1 \%)$ & $54(67.5 \%)$ & \multirow{3}{*}{0.489} & \multirow{3}{*}{0.783} \\
\hline Elevated & $2(7.4 \%)$ & $6(7.5 \%)$ & & \\
\hline Hypertension & $5(18.5 \%)$ & $20(25 \%)$ & & \\
\hline
\end{tabular}

\section{DISCUSSION}

Pediatric hypertension and obesity have both been on the rise worldwide. Each is associated with increased risk of cardiovascular morbidity and mortality. Obesity and hypertension are worrisome health hazards in Kingdome of Saudi Arabia (KSA) based on their substantial prevalence among Saudi children ${ }^{(8,17)}$. Unfortunately, the knowledge about pediatric hypertension among physicians is still insufficient. The main obstacles include not only the limited knowledge, but also the difficulty of performing multiple BP measurements over the different age in years, which are essential for proper diagnosis ${ }^{(18)}$. Many guidelines were emerged for early diagnosis and management of hypertension in children, Saudi Guidelines for hypertension has been updated lately in $2018^{(10)}$. The current study was conducted not only to address the interchangeable relation between obesity and hypertension in children but also it was aimed to reveal the diagnostic implications of altering normative based thresholds in diagnosis and classification of normal or high blood pressure using fourth version of S-GL (national) versus updated version of AAP-GL (international) among overweight/obese students. We needed to understand if international students living in Jeddah might be recommended to follow either national or international guidelines ${ }^{(10)}$.

The current study included 299 students, 5 year to 17 year-old, of mixed nationalities (Saudi and non-Saudi) with different body mass index. The prevalence rate of high BMI in our study was (33.7\%); overweight (20\%) and obese students (13.7\%) versus $66.3 \%$ normal BMI students. This was matched with the prevalence of overweight and obesity found by previous Saudi study ( $23.1 \%$ and $9.3 \%$, respectively) (19). The percent of high BMI students were significantly higher among adolescents (above 13 year) similar to other Saudi studies ${ }^{(20)}$. Female students were the predominate in overweight group while male students were the majority among the obese. This was supported by many studies. ${ }^{(21,22)}$.

Although all participants were asymptomatic, the prevalence of hypertension in the current study was (27.4\%), near to international studies carried over school-aged children at USA (19.4\%) and Italy, 22.2\% $(23,24)$. This declared the global trend of increasing prevalence of childhood hypertension during the past decades.

In contrast, the current prevalence rate of hypertension and Pre-HTN (29.5\%) were higher than reported by an Egyptian study Abu El-Foutoh $\boldsymbol{e t}$ al. in which P-HTN: $5.7 \%$ and HTN: $4.0 \%$, at 2011 as the obesity prevalence was $10.3 \%{ }^{(25)}$. Whereas another Egyptian study at 2016 had reported significant rise in the prevalence of hypertension to $15.4 \%$ when the obesity prevalence was $20 \%{ }^{(26)}$. In addition to a metanalysis study in Africa reported also revealed less figures $5.5 \%, 12.7 \%$ over different ethnic group ${ }^{(27)}$. 
On the other side, the prevalence of HTN and PreHTN in our study were less than El Qahtani Study in 2015 at eastern province of Saudi Arabia (46\%, 55\%, respectively). Our study reported lower prevalence of systolic hypertension than reported by El Qahtani Study (19.9\% versus 29\%) and lower prevalence of diastolic hypertension (19.1\% versus 33\%). This may be attributed to different age group as El Qahtani study was conducted over students between 13-18 years old (16). In addition to different method of BP analysis; based on Saudi guidelines for the adult classification of hypertension and old nomograms. Indeed, the discrepancies in the prevalence rates of high BP can could be attributed to many risk factors BMI, genetic factors, gender, physical activates, smoking, regional variations and or differences in the used protocol in diagnosis and classification of hypertension.

The impact of high BMI over blood pressure was studied in the current study. The prevalence of prehypertension and hypertension among high BMI students (overweight/obese) students was 70\%; 39.2\% $\& 30.8 \%$, respectively by S-GL. Similar to a Brazilians study over overweight children (70.5\%). However, our study revealed different distribution of systolic hypertension (31.2\%), diastolic hypertension (18\%) and both $(19.6 \%)$, respectively when compared to Brazilian study $\left(6 \%, 33 \%\right.$ and $31.5 \%$, respectively) ${ }^{(28)}$. An Indian study was conducted over high BMI children between 5y and 10 year had reported lower prevalence of pre-HTN: (18.7\%) but it matched our prevalence of hypertension $(30.7 \%){ }^{(29)}$. The prevalence of hypertension is consistent with national publications, Saudi-GL (11\%-33\%) among obese group and 4\%-14 among overweight ${ }^{(10)}$. Our study reported increased risk for Pre-HTN\& HTN two to three times among high BMI children which is in agreement with Caribbean study at 2012 showed the odds for hypertension were 2.1 (95\% CI 1.4-3.0) for overweight children and 7.2 (95\% CI: 5.0-10.3) for obese children. ${ }^{(30)}$.

On the other side, a Saudi study conducted in Hail had reported a lower prevalence of hypertension (26.7\%) among overweight/ obese adolescents than in Jeddah $(30.8 \%)^{(31)}$. This may be attributed to the regional variations within the same country. It could be explained mainly to the improvement of the lifestyle as explained by Chinese study conducted to evaluate the blood pressure measurement of children over 20 years (1991 till 2011) at eastern, central and western regions and was recommended by El Qahtani Study too ${ }^{(16,27,32)}$. Additionally, our study revealed that obesity had positive impact on elevation of systolic measurements rather than diastolic measurement of BP. This was consistent with the findings of other studies; systolic hypertension was distributed among $52.5 \%$ of obese children ${ }^{(33)}$. The suggested mechanisms of systolic hypertension in obesity might be due to hyperactivity of the sympathetic nervous system or related to vascular dysfunction and or dyslipidemia which is in need further studies for the cause.

It was very interesting, studying the relation of two guidelines used for classification of hypertension in children: Saudi (national) and American (international) Guidelines. We were motivated to cover that part in our study to address the similarities and differences between both guidelines in estimation the prevalence of the disease among overweight/obese children of mixed nationalities living in the same country.

In agreement of new AAP-GL to Saudi-GL, the overall normotensive prevalence was similar by both guidelines and the diastolic hypertension grade I by $\mathrm{S}$ GL was slightly higher than APP-GL.

On contrary, the prevalence of systolic hypertension grade I by Saudi Guidelines was increased $(21.5 \%)$ when compared to AAP (12.5\%). Also, the prevalence of elevated systolic blood pressure by AAP-GL was higher (37.5\%) when compared to Saudi Guidelines (25.5\%). This increase in elevated blood pressure could be explained by the differential classification changes in diagnosis of hypertension between younger and older children above the age of 13 years. In addition to altering normative based thresholds of hypertension to a static threshold of 130/80 $\mathrm{mm} \mathrm{Hg}$ in children $>13$ year by new AAP-GL. Thus, resulted in a wider range of elevated BP for those children, therefore, significantly more percent with elevated BP but less hypertension. This consistent with findings reported by Bell et al and Sharma et al ${ }^{(4,34)}$. Our study evaluated the impact of static threshold of hypertension among high BMI students above age of 13 year. We found an increased prevalence of elevated BP by APP (39.5\%) and less hypertension in comparison to recorded prevalence by S-GL. Unfortunately, reclassification of teenagers $(>13 y)$ by AAP-GL shared in downregulation of hypertension from stage 1 hypertension by S-GL to elevated BP. That matched previous studies ${ }^{(4,34)}$. The Saudi Guidelines of hypertension in children might be more protective as tool for early detection of hypertension in children specially because the management plan for every class is different. Further studies are highly recommended to evaluate the possibility of undervaluation or overdiagnosis of each Guideline.

In fact, the prevalence of hypertension among obese students did not differed dramatically by age, sex, or nationality when applying the same guidelines. Among three different age groups no significant difference was found except for adolescent ( $>13$ year) in which the diastolic pre-hypertension was the least 
percent. However Our findings disagree with other studies which concluded that adolescent reported the highest prevalence of pre-HTV and HTN especially among those aged 13 to $14^{\left({ }^{(3)}\right.}$. In our results females showed higher percent for HTN and pre-HTN by S-GL compared to males but without significant difference which comes in agreement with others many studies $(17,32,33)$. The combined prevalence of prehypertension and hypertension in obese adolescents in our study was $53.6 \%$ for boys and $58.9 \%$ for girl, much greater than reported by McNiec study (30\% for males and $23 \%-30 \%$ for females, respectively) ${ }^{(35)}$. Lastly, our study revealed higher prevalence HTN and Pre- among obese Saudi students compared to Non-Saudi obese students but without significant difference and this recommended for further research.

There is a high recommendation for tracking hypertension among obese children of all nationalities by applying the most safe and protective Guidelines of hypertension in children.

\section{CONCLUSION}

Prevalence of prehypertension and hypertension are two to three times more likely to occur among obese children rather than normal. However, Saudi-GL recorded higher prevalence of grade I systolic hypertension among obese students in contrast to AAP-GL which recorded higher prevalence of elevated systolic blood pressure. Static threshold for hypertension $(130 / 80 \mathrm{mmHg}$ ) for adolescents in new APP-GL created wide gap for elevated BP that might down-regulate the diagnosis of hypertension.

\section{RECOMMENDATIONS}

Further studies are recommended especially on adolescents to differentiate the vascular changes in prehypertension states from hypertension as a reflection of static threshold which was a limitation in this study in order to realize undervaluation or overdiagnosis of hypertension.

\section{Author Declarations}

$\diamond \quad$ Conflict of Interest: The authors declare that they have no conflict of interest.

$\diamond \quad$ Consent for publication: Not applicable

$\diamond \quad$ Availability of data and materials: The data are available.

$\diamond$ Funding: Not applicable /There is no funding source.

\section{ACKNOWLEDGEMENT}

The authors would particularly like to thank the pediatrician at East Jeddah and Al-Azizyha hospitals for their cooperation during data collection. The authors acknowledge the school directors and school advocatory for their effort to support this work with special thanks to parents of the children who participated in the study.

\section{REFERENCES}

1.Collaborators GBDCRD (2017): Global, regional, and national deaths, prevalence, disability-adjusted life years, and years lived with disability for chronic obstructive pulmonary disease and asthma, 1990-2015: a systematic analysis for the Global Burden of Disease Study 2015. Lancet Respir Med.,5(9):691-706.

2.Mills K, Bundy J, Kelly Tet al. (2016): Global Disparities of Hypertension Prevalence and Control: A Systematic Analysis of Population-Based Studies From 90 Countries. Circulation, 134(6):441-50.

3.Wuhl E (2019): Hypertension in childhood obesity. Acta Paediatr.,108(1):37-43.

4.Bell C, Samuel J, Samuels J (2019): Prevalence of Hypertension in Children. Hypertension,73(1):148-52.

5.McEniery C, Cockcroft J, Roman M et al. (2014): Central blood pressure: current evidence and clinical importance. Eur Heart J.,35(26):1719-25.

6.Harris K, Benoit G, Dionne J et al. (2016): Hypertension Canada's 2016 Canadian Hypertension Education Program Guidelines for Blood Pressure Measurement, Diagnosis, and Assessment of Risk of Pediatric Hypertension. Can J Cardiol.,32(5):589-97.

7.Kelly R, Magnussen C, Sabin M et al. (2015): Development of hypertension in overweight adolescents: a review. Adolesc Health Med Ther.,6:171-87.

8.Al-Hussaini A, Bashir M, Khormi M et al. (2019): Overweight and obesity among Saudi children and adolescents: Where do we stand today? Saudi J Gastroenterol.,25(4):229-35.

9.Walter L (2018): Obesity and Central Blood Pressure in Children and Adolescents. Am J Hypertens.,31(12):12667.

10.Society SHMS (2018): Saudi Hypertension Guidelines . 4th ed. Riyadh, KSA. Saudi Hypertention Guideline 2017SLIDELEGEND.com

11.Flynn J, Kaelber D, Baker-Smith C et al. (2017): Clinical Practice Guideline for Screening and Management of High Blood Pressure in Children and Adolescents. Pediatrics, 140:3.

12.Mazor-Aronovitch K, Lotan D, Modan-Moses D et al. (2014): Blood pressure in obese and overweight children and adolescents. Isr Med Assoc J.,16(3):157-61.

13.Faul F, Erdfelder E, Lang A et al. (2007): G*Power 3: a flexible statistical power analysis program for the social, behavioral, and biomedical sciences. Behav Res Methods,39(2):175-91.

14. WHO(1995): Physical status: the use and interpretation of anthropometry. Report of a WHO Expert Committee. http://helid.digicollection.org/en/d/Jh0211e/

15. National High Blood Pressure Education Program Working Group on High Blood Pressure in C, Adolescents (2004): The fourth report on the diagnosis, evaluation, and treatment of high blood pressure in children and adolescents. Pediatrics.114(2 Suppl 4th Report):555-76.

16.Alkahtani S (2015): Pediatric hypertension in the Eastern Province of Saudi Arabia. Saudi Med J.,36(6):713-9. 
17.Abolfotouh M, Al-Alwan I, Al-Rowaily M (2012): Prevalence of Metabolic Abnormalities and Association with Obesity among Saudi College Students. Int J Hypertens., 2012:819726.

18.Hansen M, Gunn P, Kaelber D (2007): Underdiagnosis of hypertension in children and adolescents. JAMA.,298(8):874-9.

19.El Mouzan M, Foster P, Al Herbish A et al. (2010): Prevalence of overweight and obesity in Saudi children and adolescents. Ann Saudi Med.,30(3):203-8.

20.Al-Almaie S (2005): Prevalence of obesity and overweight among Saudi adolescents in Eastern Saudi Arabia. Saudi Med J.,26(4):607-11.

21.Al-Rukban M (2003): Obesity among Saudi male adolescents in Riyadh, Saudi Arabia. Saudi Med J.,24(1):27-33.

22.Washi S, Ageib M(2010): Poor diet quality and food habits are related to impaired nutritional status in 13- to 18year-old adolescents in Jeddah. Nutr Res.,30(8):527-34.

23.Sorof J, Lai D, Turner J et al. (2004): Overweight, ethnicity, and the prevalence of hypertension in schoolaged children. Pediatrics, 113(3 Pt 1):475-82.

24.Dulskiene V, Kuciene R, Medzioniene J et al. (2014): Association between obesity and high blood pressure among Lithuanian adolescents: a cross-sectional study. Ital J Pediatr.,40:102.

25.Abolfotouh M, Sallam S, Mohammed M et al. (2011): Prevalence of elevated blood pressure and association with obesity in egyptian school adolescents. Int J Hypertens.,2011:952537.

26.Allam H, Ghannam S et al. ( 2016): The prevalence of hypertension in school going children of Cairo, Egypt. $j$. ournal of Innovations in Pharmaceutical and Biological Sciences,3(4):1-5.
27.Noubiap J, Essouma M, Bigna J et al. (2017): Prevalence of elevated blood pressure in children and adolescents in Africa: a systematic review and metaanalysis. Lancet Public Health,2(8):e375-e86.

28.Noronha J, Ramos A (2012): High Blood Pressure In Overweight Children And Adolescents. Journal of Human Growth and Development,22(2):196-201.

29.Das M, Seiblal A (2017): Prevalence of hypertension in urban school children aged 5 to 10 years in North India. International Journal of Contemporary Pediatrics,4(6):2055-9.

30.Schwiebbe L, Talma H, Renders C, et al. (2012): High prevalence of hypertension in obese children in the Caribbean. Paediatr Int Child Health,32(4):204-7.

31.Ghamri R, Hegazy A, Azizkhan A et al. (2019): High blood pressure in children attending pediatric clinic at King Abdulaziz University Hospital, Jeddah, Saudi Arabia. J Family Community Med.,26(3):193-8.

32.Fan $Z$, Liao $Z$, Zong $X$ et al. (2019): Differences in prevalence of prehypertension and hypertension in children and adolescents in the eastern, central and western regions of China from 1991-2011 and the associated risk factors. PLoS One,14(1):e0210591.

33.Al-Agha A, Mahjoub A (2018): Impact of body mass index on high blood pressure among obese children in the western region of Saudi Arabia. Saudi Med J.,39(1):45-51.

34.Ferreira J, Aydos R (2010): [Prevalence of hypertension among obese children and adolescents]. Cien Saude Colet.,15(1):97-104.

35.McNiece K, Poffenbarger T, Turner J et al. (2007): Prevalence of hypertension and pre-hypertension among adolescents. J Pediatr.,150(6):640-4, 4 e1. 